\title{
Transmission electron microscopy studies of the zona reaction in pig oocytes fertilized in vivo and in vitro
}

\author{
H. Funahashi ${ }^{1,3}$, H. Ekwall2, K. Kikuchi ${ }^{1 *}$ and \\ H. Rodriguez-Martinez ${ }^{1}$ \\ Departments of ${ }^{1}$ Obstetrics and Gynaecology and ${ }^{2}$ Anatomy and Histology, Faculty of \\ Veterinary Medicine, Swedish University of Agricultural Sciences (SLU), SE-750 07 Uppsala, \\ Sweden; and ${ }^{3}$ Department of Animal Science, Faculty of Agriculture, Okayama University, \\ Okayama 700-8530, Japan
}

The aim of this study was to determine the ultrastructure of cross-sectioned zonae pellucidae of in vitro-matured and ovulated pig oocytes before or after sperm penetration in vitro and in vivo, respectively. The in vitro and in vivo (ovulated) oocytes and zygotes (fertilized in vitro and in vivo) were fixed with glutaraldehyde either directly or after pretreatment with ruthenium red and saponin, processed and then examined using transmission electron microscopy. The thickness of the zona pellucida, as measured on the section of the specimens with largest diameter fixed with glutaraldehyde, differed between the in vivo $(9.19 \pm 0.47 \mu \mathrm{m})$ and in vitro $(5.95 \pm$ $0.51 \mu \mathrm{m})$ oocytes. The in vivo oocytes had a rather thick external mesh-like structure, whereas it was much thinner in the in vitro oocytes. This mesh-like external rim was less apparent in both in vivo and in vitro zygotes. Obvious differences in the density of the lattice formed by the fixed zonae pellucidae were visible between the outer and inner (ad-oolemmal) zonae. The outer area always formed a concentrically arrayed fibrillar network, whereas the inner area showed a much more compact, trabecule-like mesh. However, both areas, but particularly the outer network, were much more compacted after the zona reaction. Clear differences in the degree of fibrillar aggregation of the inner zona area were also observed between in vitro and in vivo zygotes, being much higher in the latter. This fibrillar network was more clearly visible in the zygotes pretreated with ruthenium red and saponin; the in vitro zygotes had a fibrillar, radially oriented set of parallel fibrils, whereas it was much more aggregated and trabecule-like in the in vivo zygotes. These results demonstrate that the fine structure of the zona pellucida and the zona reaction at sperm penetration differ between pig oocytes fertilized in vivo and in vitro. Moreover, the ultrastructure of the outer and inner pig zonae pellucidae has a different network organization.

\section{Introduction}

The zona pellucida is a glycoprotein coat that protects oocytes and embryos from physical damage and also regulates sperm penetration. At fertilization, the entry of multiple spermatozoa into the oocyte is prevented by the zona pellucida. However, polyspermic penetration has been a major obstacle of in vitro fertilization (IVF) of in vitro-matured oocytes in pigs. Wang et al. (1998a,b, 1999) reported that the abnormally high incidence of polyspermic penetration of pig oocytes in vitro is not due to delayed or incomplete cortical granule exocytosis but more probably to a delayed zona pellucida reaction or simultaneous sperm penetration or both. Funahashi et al. (2000) demonstrated that the surface morphology of the zona pellucida and the zona reaction at sperm penetration differed between in vitro-matured and ovulated oocytes, as detected by

Email: hirofun@cc.okayama-u.ac.jp

*Present address: National Institute of Agrobiological Sciences, Genetic Diversity Department, Tsukuba, Ibaraki 305-8602, Japan scanning electron microscopy (SEM). In that study, the outer morphology of ovulated oocytes was a mesh-like structure, whereas that of in vitro-matured oocytes was much smoother. Wang et al. (1998a) reported that the resistance of the zona pellucida to dissolution by pronase was different between ovulated and in vitro-matured oocytes and Kim et al. (1996) reported that the resistance of in vitro-matured oocytes was increased by exposure to oviductal fluid. However, it has not been clarified whether the morphological differences between the zonae pellucidae of in vitromatured and ovulated pig oocytes are only at the surface and, moreover, whether the zona reaction reflects the origin of the oocytes.

In the present study, changes in the ultrastructure of cross-sectioned zonae pellucidae of in vitro-matured and ovulated (in vivo) pig oocytes were observed before or after sperm penetration in vitro and in vivo, respectively, using transmission electron microscopy (TEM). The differences in the inner structure of zonae pellucidae between in vitro and ovulated pig oocytes before and after sperm penetration are described. 


\section{Materials and Methods}

\section{Reagents}

All reagents used were purchased from Sigma Chemical Co (St Louis, MO).

\section{Media}

The medium used for the collection of cumulus-oocyte complexes (COCs) and washing was modified TL-HepesPVA medium composed of $114 \mathrm{mmol} \mathrm{NaCl} \mathrm{I-1}, 3.2 \mathrm{mmol}$ $\mathrm{KCl} \mathrm{I}-1,2 \mathrm{mmol} \mathrm{NaHCO}_{3} \mathrm{I}^{-1}, 0.34 \mathrm{mmol} \mathrm{KH}_{2} \mathrm{PO}_{4} \mathrm{I}^{-1}$, $10 \mathrm{mmol}$ sodium lactate $\mathrm{I}^{-1}, 0.5 \mathrm{mmol} \mathrm{MgCl}_{2} \cdot 6 \mathrm{H}_{2} \mathrm{O} \mathrm{I}^{-1}$, $2 \mathrm{mmol} \mathrm{CaCl} 2 \cdot 2 \mathrm{H}_{2} \mathrm{Ol}^{-1}, 10 \mathrm{mmol}$ Hepes $\mathrm{I}^{-1}, 0.2 \mathrm{mmol}$ sodium pyruvate $\mathrm{I}^{-1}, 12 \mathrm{mmol}$ sorbitol $\mathrm{I}^{-1}, 0.1 \%(\mathrm{w} / \mathrm{v})$ polyvinylalcohol, $25 \mu \mathrm{g}$ gentamicin $\mathrm{ml}^{-1}$ and $65 \mu \mathrm{g}$ potassium penicillin $\mathrm{G} \mathrm{ml}^{-1}$. The basic maturation medium (OMM37) used was BSA-free North Carolina State University 37 medium (Petters and Wells, 1993) supplemented with 0.6 mmol cysteine $\mathrm{I}^{-1}, 5 \mu \mathrm{g}$ insulin $\mathrm{ml}^{-1}$ and $10 \%(\mathrm{v} / \mathrm{v})$ pig follicular fluid (Funahashi et al., 1997). The basic medium used for IVF was modified BO solution (Brackett and Oliphant, 1975), composed of $112.0 \mathrm{mmol} \mathrm{NaCl} \mathrm{I}^{-1}$, $4.02 \mathrm{mmol} \mathrm{KCl} \mathrm{I}^{-1}, 37.0 \mathrm{mmol} \mathrm{NaHCO}_{3} \mathrm{I}^{-1}, 0.83 \mathrm{mmol}$ $\mathrm{NaH}_{2} \mathrm{PO}_{4} \cdot 2 \mathrm{H}_{2} \mathrm{O} \mathrm{I}^{-1}, 1.25 \mathrm{mmol}$ sodium pyruvate $\mathrm{I}^{-1}$, $0.50 \mathrm{mmol} \mathrm{MgCl}_{2} \cdot 6 \mathrm{H}_{2} \mathrm{O} \mathrm{I}^{-1}, 7.50 \mathrm{mmol} \mathrm{CaCl} \cdot 2 \mathrm{H}_{2} \mathrm{Ol}^{-1}$, $50 \mu$ g gentamicin $\mathrm{ml}^{-1}, 6 \mathrm{mg} \mathrm{BSA} \mathrm{ml}^{-1}$ (A6003; Sigma) and $5 \mathrm{mmol}$ caffeine sodium benzoate $\mathrm{I}^{-1}$. All media were equilibrated at $38.5^{\circ} \mathrm{C}$ in an atmosphere of $5 \% \mathrm{CO}_{2}$ in air overnight before incubation of the oocytes. Pig follicular fluid was prepared from antral follicles $(3-6 \mathrm{~mm}$ in diameter) as described by Funahashi et al. (1994).

\section{Collection of ovulated oocytes and zygotes matured and fertilized in vivo}

Immediately after weaning, multiparous crossbred (Yorkshire $\times$ Landrace) sows $(n=11)$ were put into individual pens and checked twice a day for signs of oestrus in the presence of a boar. Ovulated oocytes and resulting zygotes were collected surgically with TL-Hepes-PVA at 44-48 h after detection of standing oestrus from control and inseminated sows, respectively. Only eggs with many spermatozoa binding to the zona pellucida (as observed by microscopy) were selected and considered as zygotes. Ovulated oocytes and zygotes were washed three times with an enzyme inhibitor solution (modified TL-Hepes-PVA supplemented with $1 \mathrm{mg}$ EDTA ml-1, $10 \mu \mathrm{g}$ lima bean trypsin inhibitor $\mathrm{I}^{-1}$ and $0.1 \mathrm{mmol}$ phenylmethylsulphonyl fluoride ${ }^{-1}$ ) and used for the experiment.

\section{Preparation of in vitro-matured oocytes and zygotes matured and fertilized in vitro}

Ovaries were collected from prepubertal gilts at a local abattoir. The ovaries were transported to the laboratory at $23-27^{\circ} \mathrm{C}$. COCs were aspirated through an 18-gauge needle into a disposable $10 \mathrm{ml}$ syringe from antral follicles
(3-6 $\mathrm{mm}$ in diameter) on the surface of ovaries, washed three times with modified TL-Hepes-PVA medium, and then collected in $3 \mathrm{ml}$ of fresh modified TL-Hepes-PVA medium. COCs with uniform ooplasm and a compact cumulus cell mass $(n=50)$ were washed three times with OMM37 supplemented with $1 \mathrm{mmol}$ dibutyryl cAMP I-1, $0.5 \mu \mathrm{g} \mathrm{FSH} \mathrm{ml}{ }^{-1}$ and $0.5 \mu \mathrm{g} \mathrm{LH} \mathrm{ml}^{-1}$, and subsequently cultured in $500 \mu \mathrm{l}$ of the same medium covered with paraffin oil for $20 \mathrm{~h}$ at $38.5^{\circ} \mathrm{C}$ in an atmosphere of $5 \% \mathrm{CO}_{2}$ in air. The complexes were transferred to $500 \mu \mathrm{l}$ OMM37 (without dibutyryl cAMP, FSH and $\mathrm{LH}$ ) after washing three times with the same medium. The complexes were cultured for an additional $24 \mathrm{~h}$ (Funahashi and Day, 1993; Funahashi et al., 1994). After culture, oocytes were stripped of cumulus cells by pipetting with $0.1 \%(\mathrm{w} / \mathrm{v})$ hyaluronidase and washed carefully three times with modified BO medium.

Frozen-thawed boar sperm suspensions were washed three times with Dulbecco's PBS supplemented with $0.1 \%$ $(\mathrm{w} / \mathrm{v})$ BSA by centrifugation at $1500 \mathrm{~g}$ for $4 \mathrm{~min}$ each time. The pellets containing spermatozoa were resuspended at $2 \times 10^{6}$ cells ml-1 in modified BO medium and used for IVF. Denuded oocytes $(n=30)$ were cocultured with $1 \times 10^{6}$ cells $\mathrm{ml}^{-1}$ in a $100 \mu \mathrm{l}$ droplet of modified BO medium under paraffin oil for $10 \mathrm{~h}$ at $38.5^{\circ} \mathrm{C}$ in an atmosphere of $5 \% \mathrm{CO}_{2}$ in air. At $10 \mathrm{~h}$ after the start of coculture, in vitro-matured and fertilized (IVM-IVF) eggs were centrifuged in an enzyme inhibitor solution at $15000 \mathbf{g}$ for $3 \mathrm{~min}$. Only eggs containing two polar bodies and at least two pronuclei were selected under a microscope and used for the experiment. Specimens of in vitro-matured oocytes and IVM-IVF ova were collected from four cycles of in vitro maturation and fertilization.

\section{Sample preparation and observation by transmission electron microscopy}

In vivo-matured oocytes (ovulated oocytes) and in vivo zygotes, as well as the in vitro-matured oocytes and IVM-IVF ova with two polar bodies and at least two pronuclei (in vitro zygotes) were fixed in $1 \%(\mathrm{v} / \mathrm{v})$ glutaraldehyde solution in $0.1 \mathrm{~mol}$ cacodylate buffer $\mathrm{I}^{-1}$ $(\mathrm{pH} 7.2)$ and stored in the same solution at $4^{\circ} \mathrm{C}$ until processed. Some of the samples were incubated in a solution of $1 \%(\mathrm{w} / \mathrm{v})$ ruthenium red and $0.02 \%(\mathrm{w} / \mathrm{v})$ saponin in $0.1 \mathrm{~mol}$ cacodylate buffer $\mathrm{I}^{-1}$ and fixed in $1 \%(\mathrm{v} / \mathrm{v})$

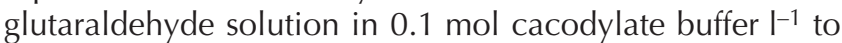
increase the likelihood of disclosing the fibrillar networks of the zonae pellucidae (Familiari et al., 1992). After postfixation for $5 \mathrm{~min}$ in $2 \%(\mathrm{w} / \mathrm{v})$ osmium tetroxide in the same buffer, continuous sections of oocytes and zygotes were processed conventionally for TEM. Therefore, zona pellucida thickness of the specimen with the largest diameter should represent the correct thickness of the zona pellucida. The thickness should be greater in specimens with smaller diameter. Counter-stained ultrathin sections with a maximum diameter of specimen were examined in a Philips 420 transmission electron microscope at $80 \mathrm{kV}$. 


\section{Statistical analysis}

Statistical analyses of zona pellucida thickness using data from at least three sows or four replicate trials were carried out by ANOVA and Fisher's protected least significant difference test using the STATVIEW program (Abacus Concepts Inc., Berkeley, CA) to determine treatment differences. Data are expressed as mean \pm SEM. The level of significance was $P<0.05$.

\section{Results}

In images retrieved via TEM, the mean thickness of zona pellucida samples was greater $(P<0.01)$ in ovulated oocytes (9.19 $\pm 0.47 \mu \mathrm{m} ; n=9$ samples from five sows) than in in vitro-matured oocytes $(5.95 \pm 0.51 \mu \mathrm{m} ; n=11$ samples from four replicates; Fig. 1). The thickness of the zona pellucida was greater $(P<0.01)$ in in vivo zygotes (16.04 $\pm 1.80 \mu \mathrm{m} ; n=6$ samples from three sows) than in ovulated oocytes, whereas the thickness of the zona pellucida of in vitro zygotes $(5.51 \pm 0.24 \mu \mathrm{m} ; n=14$ samples from four replicates) did not differ from that of in vitro-matured oocytes (Fig. 1). The ovulated oocytes had a rather thick external mesh-like structure (about one-third of the zona pellucida; Fig. 2a,b), whereas the structure was much thinner in the in vitro-matured oocytes (Fig. 3a,b). This mesh-like external rim was less apparent in both in vivo and in vitro zygotes. More remnants of foot processes of the corona cells were found inside the zona pellucida, especially in the external part, in in vitro-matured oocytes (Fig. 3a) and in vitro zygotes (Fig. 4a), compared with ovulated oocytes (Fig. 2a) and in vivo zygotes (Fig. 5a). As expected, cortical granules were present under the oolemma in both ovulated (Fig. 2a,c) and in vitro-matured oocytes (Fig. 3a,c) but were completely absent in in vivo (Fig. 5a,c) and in vitro (Fig. 4a,c) zygotes. In addition, zona pellucida sperm tracks were evident in the in vivo (Fig. 5b) and in vitro (Fig. 4b) zygotes. Obvious differences in the density of the lattice formed by the fixed zonae pellucidae were visible between the outer and inner (ad-oolemmal) zona pellucida. The outer area always formed a concentrically arrayed fibrillar network, mainly parallel to the surface of cytoplasm (Figs 2d and 3d), whereas the inner area showed a much more compact, trabecule-like mesh (Figs 2e and 3e). The fibrillar network in the outer zona pellucida was more coarse in ovulated oocytes (Fig. 2d) than in the in vitro-matured oocytes (Fig. 3d). Both areas, but particularly the outer network, were much more compact after the zona pellucida reaction (Figs $4 d$ and $5 d$ ). The concentrically arrayed fibrillar network was not observed in in vivo zygotes (Fig. $5 \mathrm{~d}$ ), but was visible, albeit incomplete, in in vitro zygotes (Fig. 4d). Clear differences in the degree of fibrillar aggregation of the inner zona pellucida area were observed between in vivo (Fig. 5e) and in vitro (Fig. 4e) zygotes: fibrillar aggregation was much higher in the former. This fibrillar network was more clearly visible in the zygotes pre-treated with ruthenium red and

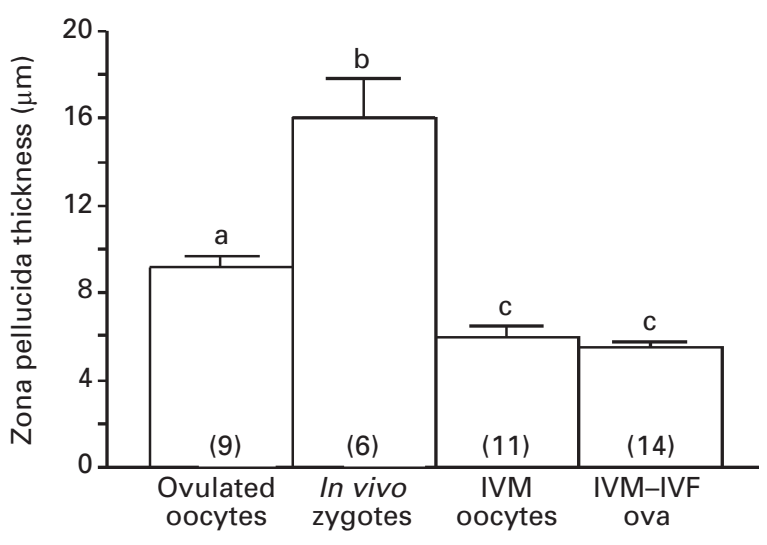

Fig. 1. Mean thickness of pig zonae pellucidae after fixation and preparation for transmission electron microscopy. In vivo zygotes: zygotes resulting from fertilization of ovulated oocytes; IVM oocytes: oocytes matured in vitro; IVM-IVF ova: zygotes resulting from in vitro maturation and fertilization. abcValues with different letters are significantly different $(P<0.05)$. Numbers in parentheses show the number of zonae pellucidae examined in each group.

saponin for a shorter period (Fig. 6 a-f). The in vitro zygotes had a conspicuous fibrillar, radially orientated set of parallel fibrils in the inner area of the zona pellucida (Fig. 6 a,c), whereas the fibrillar network in the in vivo zygotes (Fig. 6 b,d) was much more aggregated and trabecule-like (Fig. 6c,d). The outer part of the zonae pellucidae was less arranged in both cases, partially losing these orientations (Fig. 6e,f).

\section{Discussion}

A heterogeneous structure in pig zona pellucida with differences between the external and internal surface has been observed using light microscopy (Dunbar et al., 1980) and TEM (Dietl and Czuppon, 1984). The aim of the present study was to clarify whether the fine cross-sectioned structure of pig zonae pellucidae differs between ovulated and in vitro-matured oocytes, and how those structures change before and after the zona reaction. Differences in morphology were observed between the external and internal structure of the zona pellucida in both in vitromatured and ovulated oocytes. Furthermore, a rather thick external mesh-like structure was found in ovulated oocytes compared with in vitro-matured oocytes. Using SEM, Funahashi et al. (2000) observed that the outer surface of the zona pellucida of ovulated pig oocytes has a mesh-like structure with numerous fenestrations and an uneven surface with a micro-trabecular appearance, whereas the outer morphology of the zona pellucida of in vitro-matured oocytes is more compacted and smooth. The difference in external morphology of cross-sectioned zonae pellucidae observed in the present study reflects the surface structure demonstrated previously with SEM. In the present study, traces of foot processes of the corona cells were more common in the external zona pellucida in in vitro-matured 

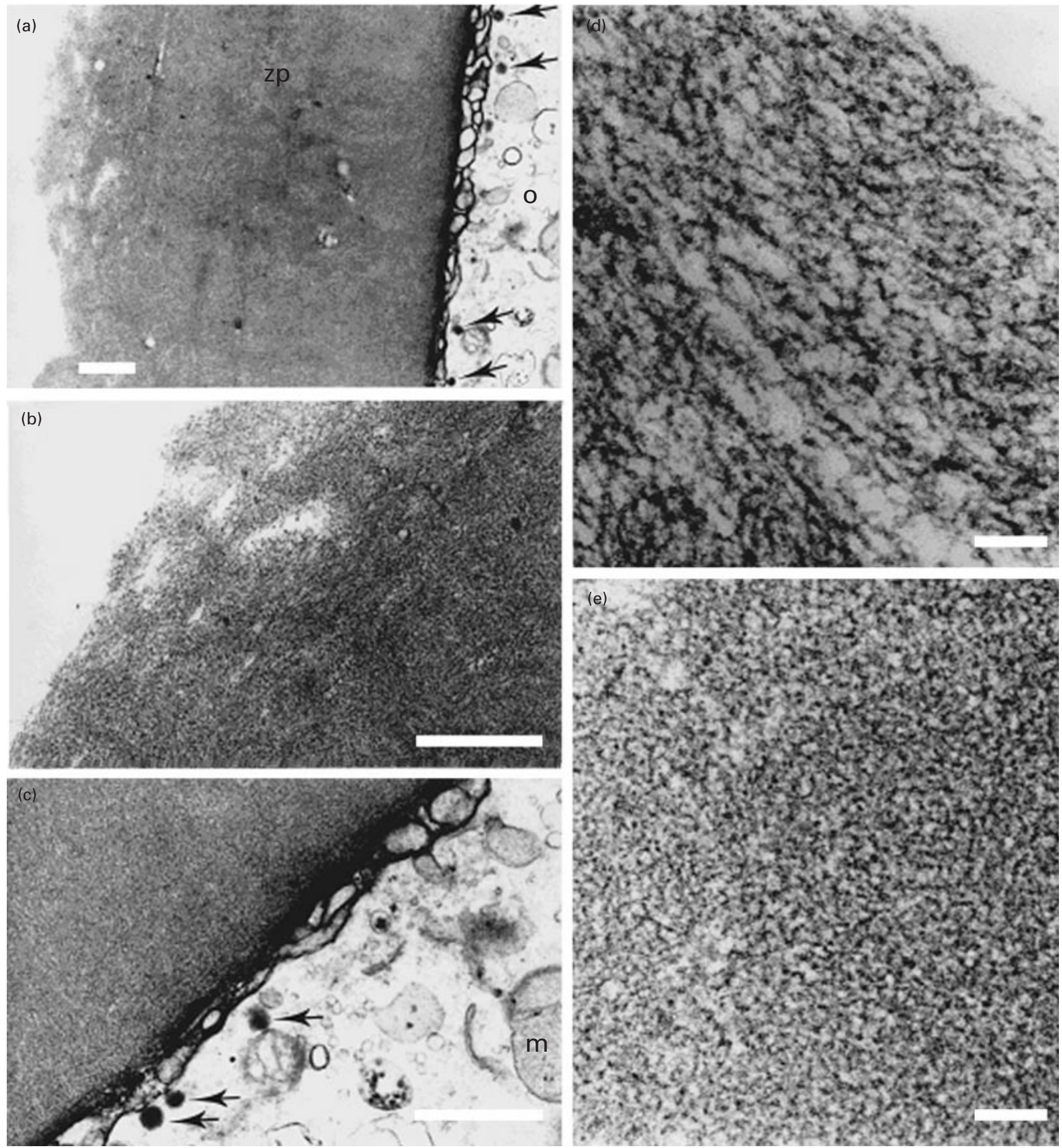

Fig. 2. Transmission electron microscope images of the zonae pellucidae of ovulated pig oocytes. (a) A part of the zona pellucida (zp). o: ooplasma. Arrows show cortical granules. (b) Outer part of the zona pellucida. (c) Inner part of zona pellucida. m: mitochondrion. Arrows show cortical granules. (d) Outer part of zona pellucida. (e) Inner part of zona pellucida. Scale bars represent $1 \mu \mathrm{m}(\mathrm{a}, \mathrm{b}, \mathrm{c})$ or $100 \mathrm{~nm}(\mathrm{~d}, \mathrm{e})$.

oocytes and in vitro zygotes compared with ovulated oocytes and zygotes. These results indicate that maturation of the zona pellucida may not have been achieved fully in the in vitro-matured oocytes, supporting our previous observation by SEM (Funahashi et al., 2000). Although current systems for in vitro production of pig embryos can result in development of $>40 \%$ of embryos to the blastocyst stage and can produce normal piglets (Funahashi and Day, 

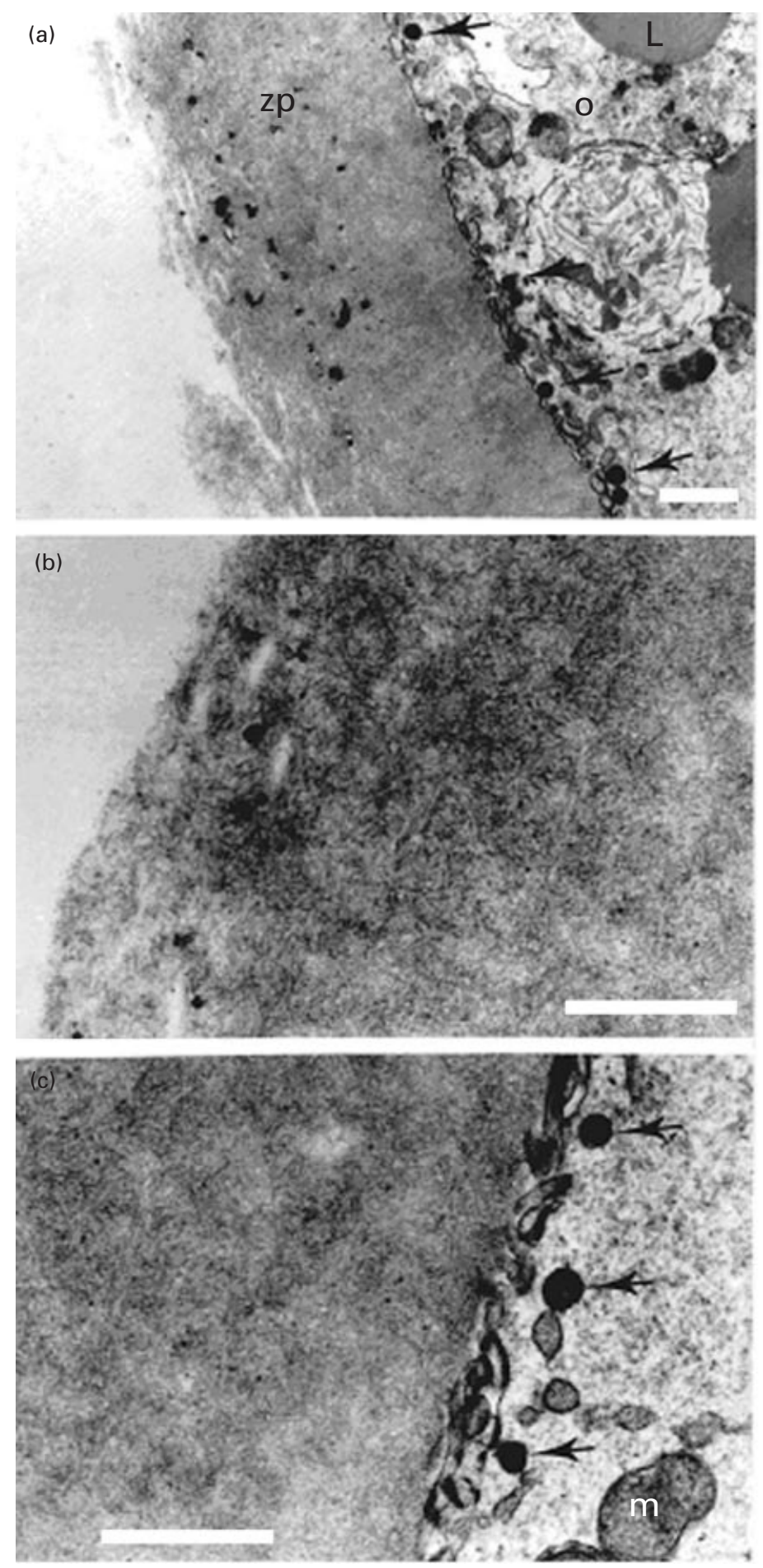
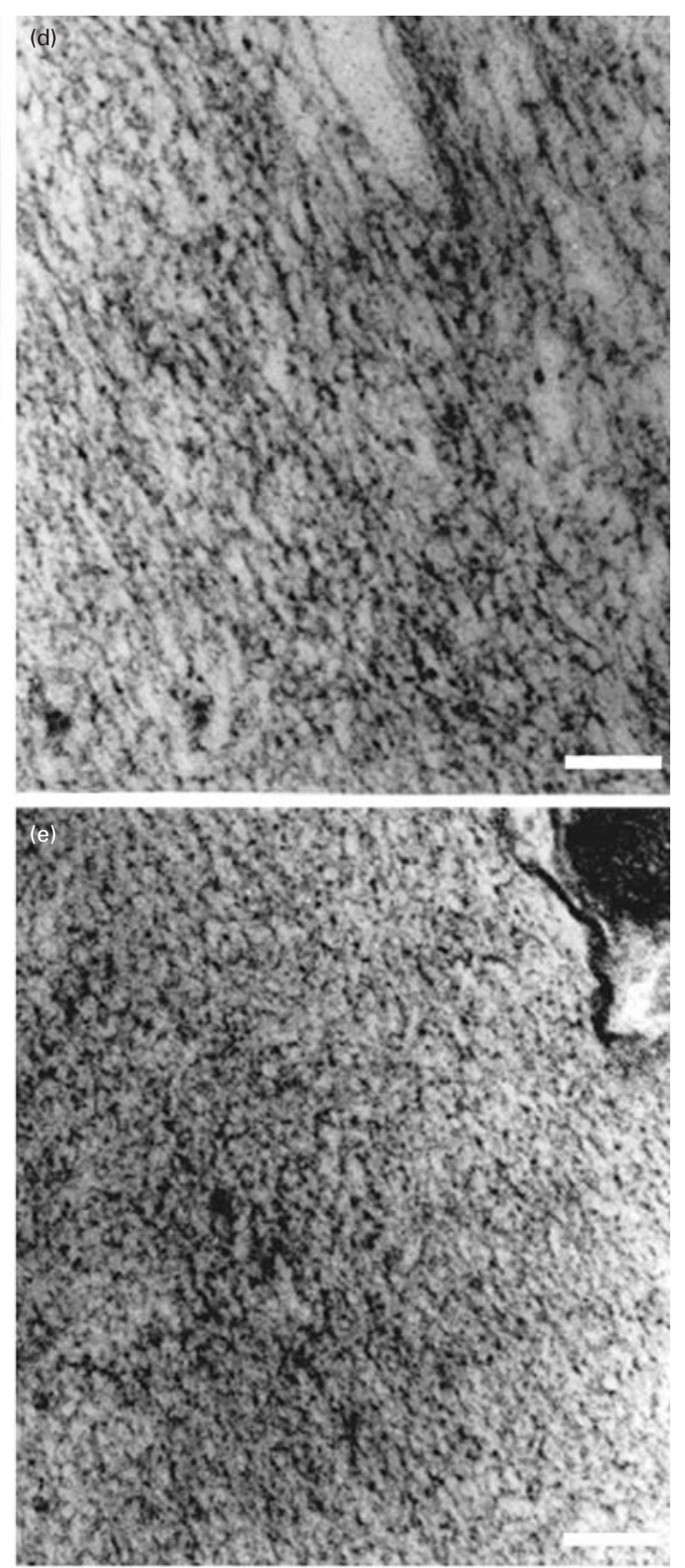

Fig. 3. Transmission electron microscope images of the zonae pellucidae of in vitro-matured pig oocytes. (a) A part of the zona pellucida (zp). o: ooplasma; L: lipid droplet. Arrows show cortical granules. (b) Outer part of zona pellucida. (c) Inner part of zona pellucida. m: mitochondrion. Arrows show cortical granules. (d) Outer part of zona pellucida. (e) Inner part of zona pellucida. Scale bars represent $1 \mu \mathrm{m}$ $(\mathrm{a}, \mathrm{b}, \mathrm{c})$ or $100 \mathrm{~nm}(\mathrm{~d}, \mathrm{e})$.

1997; Day, 2000), maturation of zona pellucida in pig oocytes still appears to be insufficient in vitro. Therefore, in vitro-matured oocytes appear to have undergone insufficient morphological maturation of zonae pellucidae.
At a high magnification, it was found that a concentrically arrayed fibrillar network, mainly parallel to the surface of cytoplasm, was always observed in the outer area of the zona pellucida in both ovulated and in vitro- 

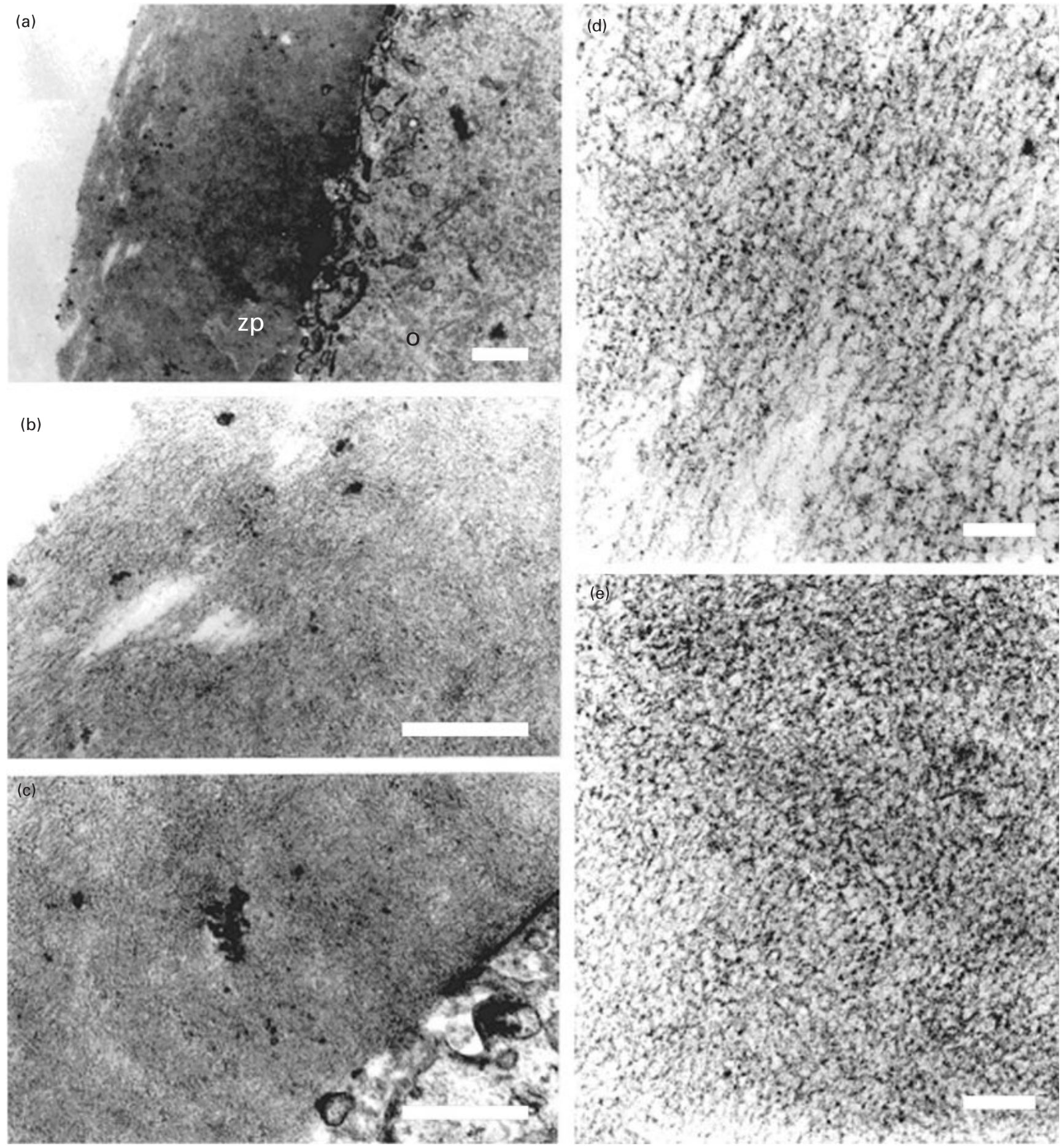

Fig. 4. Transmission electron microscope images of the zonae pellucidae of an in vitro-fertilized pig ovum. (a) A part of the zona pellucida (zp). o: ooplasma. (b) Outer part of zona pellucida. (c) Inner part of zona pellucida. (d) Outer part of zona pellucida. (e) Inner part of zona pellucida. Scale bars represent $1 \mu \mathrm{m}(\mathrm{a}, \mathrm{b}, \mathrm{c})$ or $100 \mathrm{~nm}(\mathrm{~d}, \mathrm{e})$.

matured oocytes. However, the fibrillar network was coarser in ovulated oocytes than in in vitro-matured oocytes. The zona pellucida of ovulated pig oocytes contains additional macromolecules (Hedrick et al., 1987), such as the oestrogen-dependent oviductal glycoproteins secreted into the oviductal lumen (Brown and Cheng, 1986; Buhi et al., 1993). The zona pellucida of ovulated oocytes, but not of in vitro-matured oocytes, has resistance to dissolution by pronase (Wang et al., 1998a), and it has been demonstrated that the resistance of in vitro-matured oocytes 

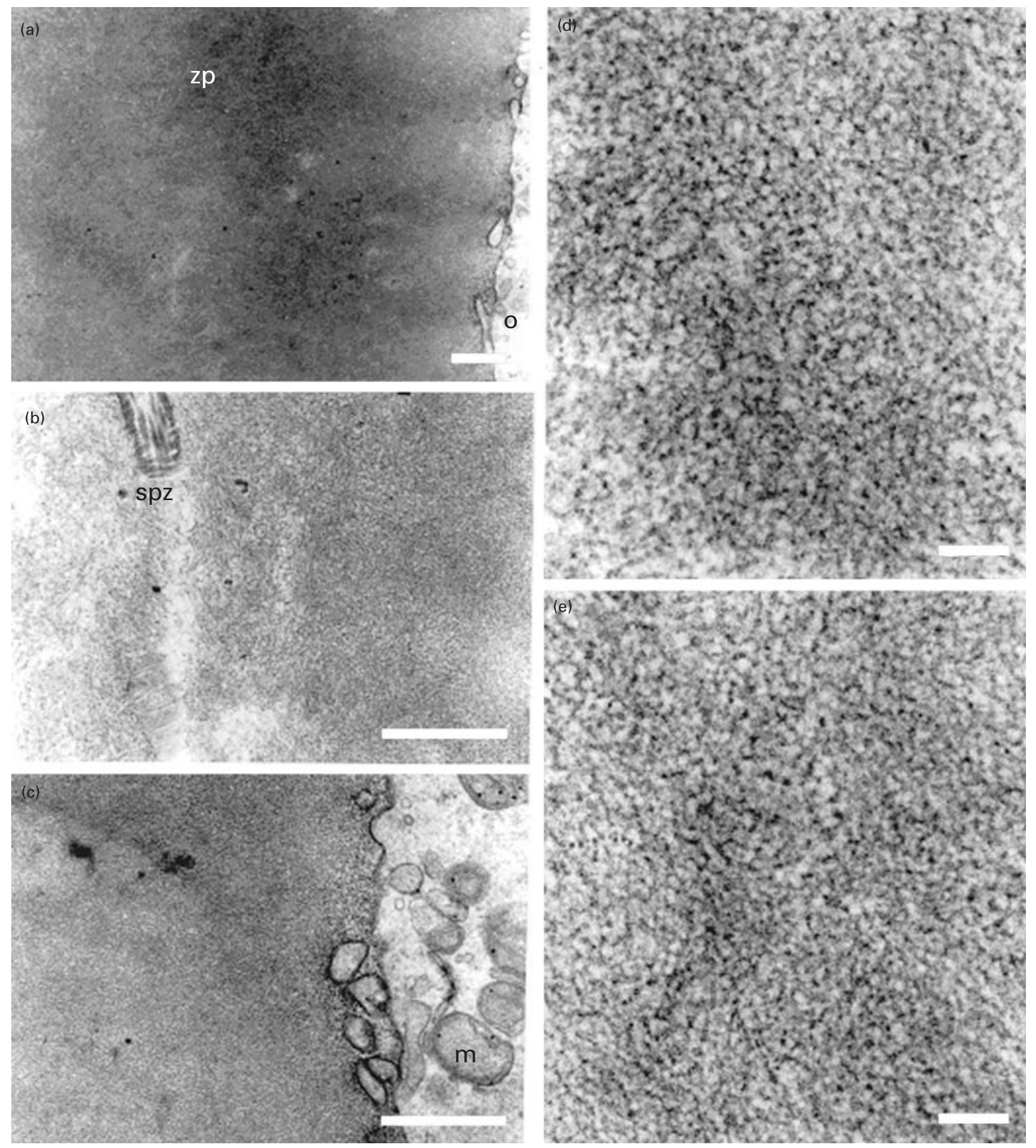

Fig. 5. Transmission electron microscope images of the zonae pellucidae of an in vivo pig zygote. (a) A part of the zona pellucida (zp). o: ooplasma. (b) Outer part of zona pellucida. spz: sperm tail. (c) Inner part of zona pellucida. m: mitochondrion. (d) Outer part of zona pellucida. (e) Inner part of zona pellucida. Scale bars represent $1 \mu \mathrm{m}(\mathrm{a}, \mathrm{b}, \mathrm{c})$ or $100 \mathrm{~nm}(\mathrm{~d}, \mathrm{e})$.

increases after exposure to oviductal fluid (Kim et al., 1996). As the resistance of ovulated oocytes was much higher in the outer surface of the zona pellucida $(\mathrm{H}$. Funahashi, unpublished), the coarse fibrillar network observed in ovulated oocytes may be the result of permeation of the oestrogen-dependent oviductal glycoproteins containing pronase inhibitors. However, aggregated fibrils in the inner zona pellucida were observed in both ovulated and in vitro- 

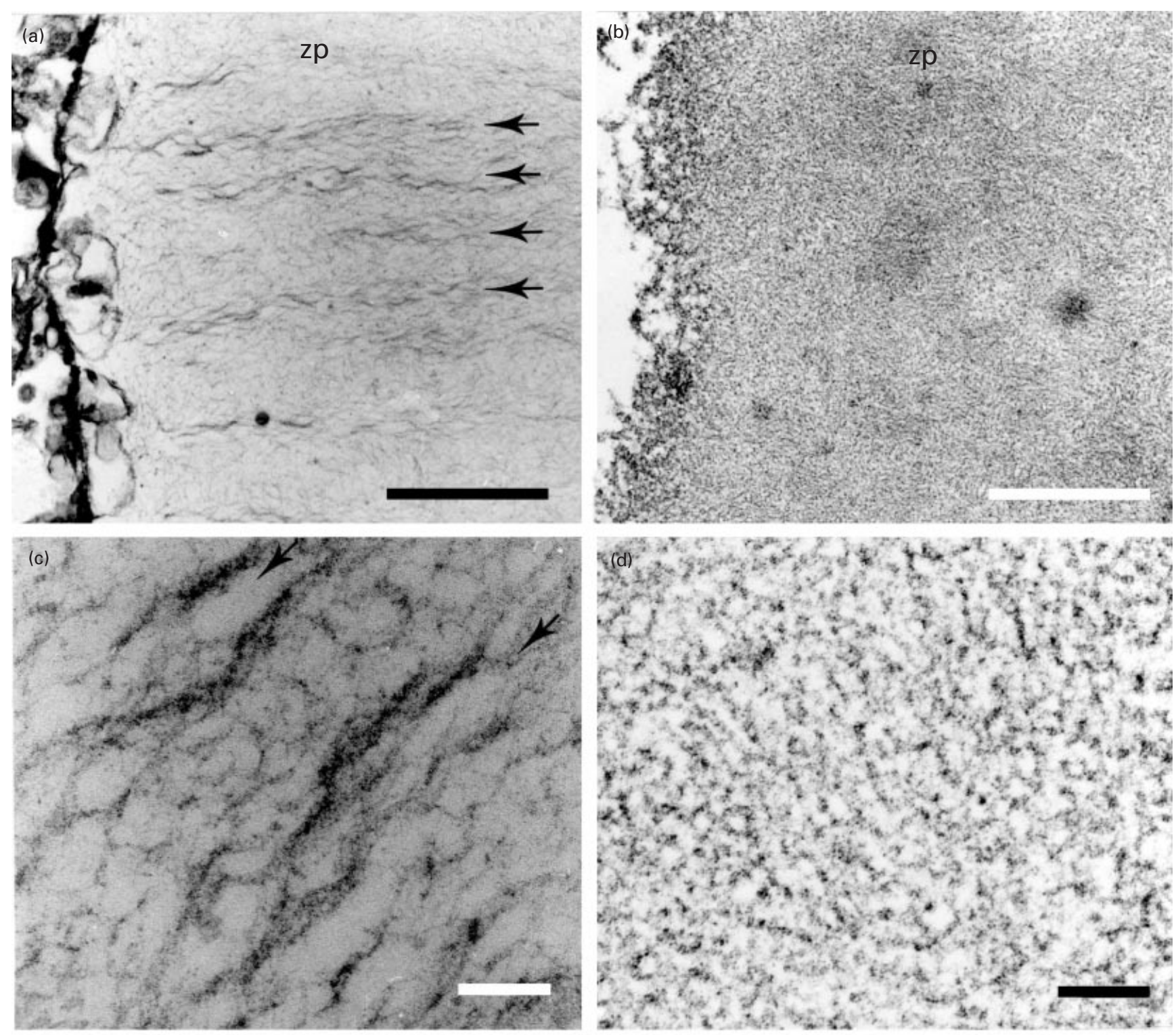

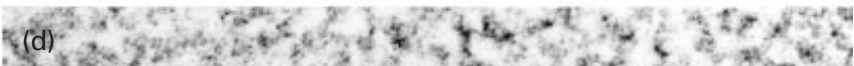

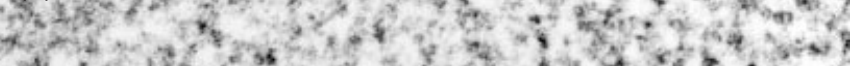

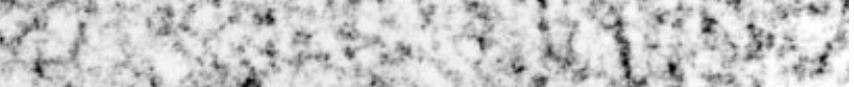

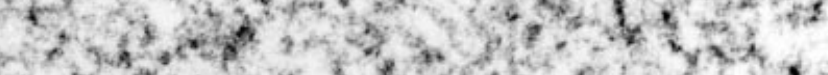

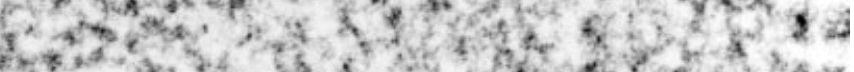

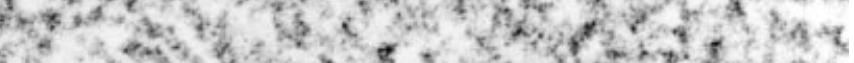

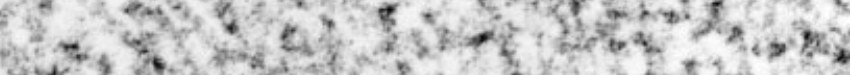

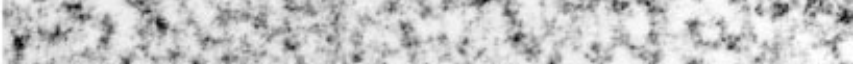

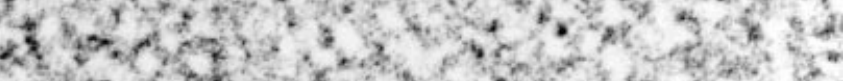

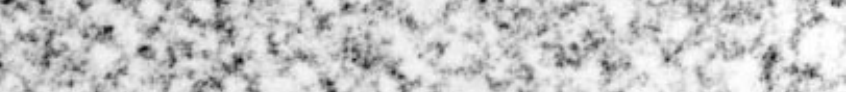

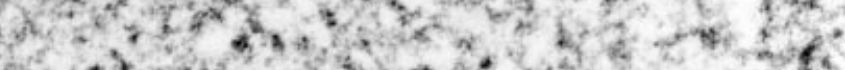

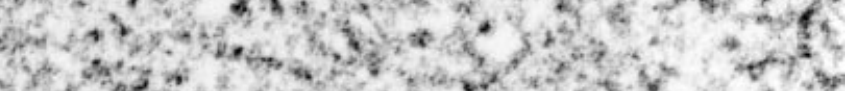

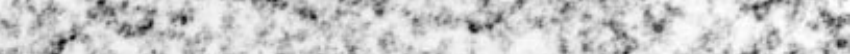

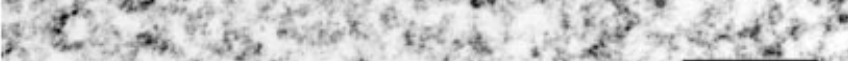

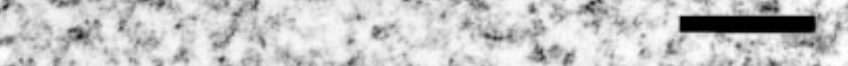

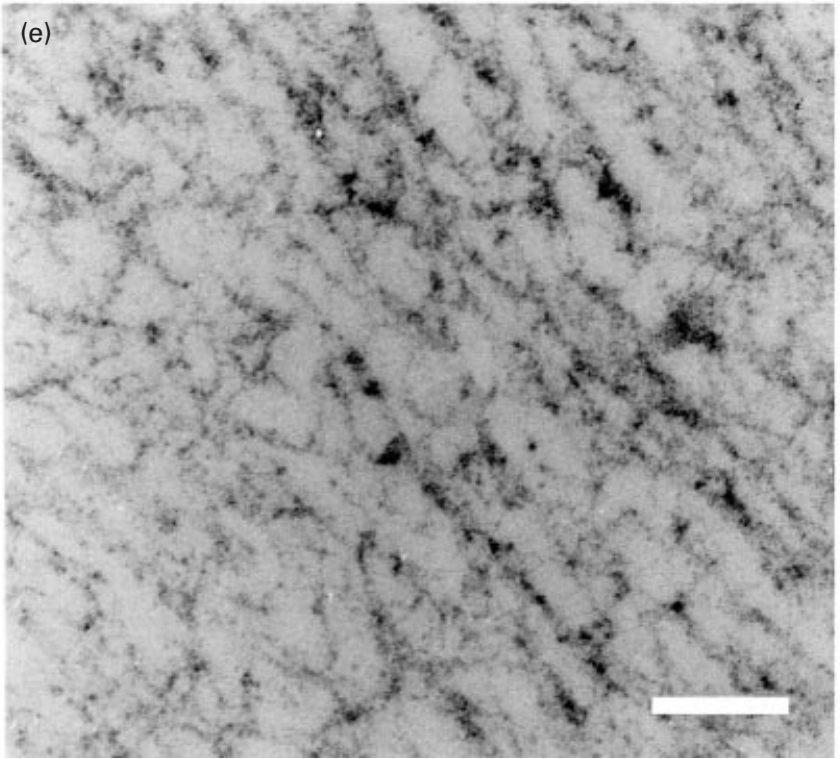

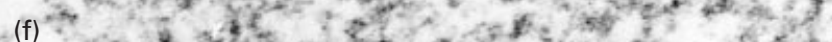

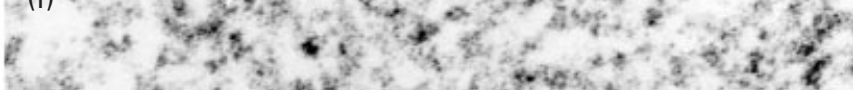

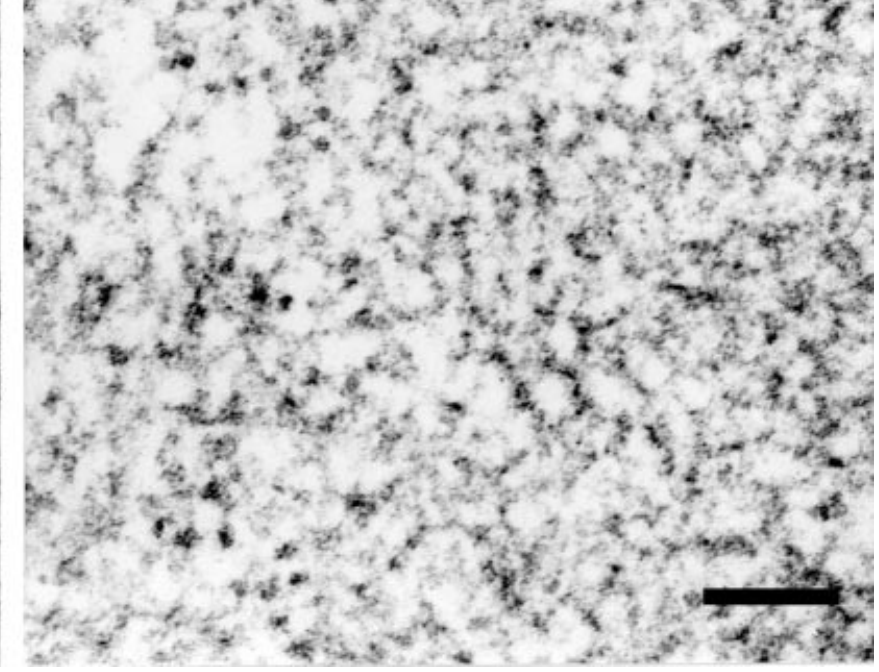


matured oocytes, although the degree of aggregation of ovulated oocytes was much higher than in in vitro-matured oocytes. Therefore, the inner structure of the zona pellucida may be stiffer than the outer surface. Furthermore, as a large number of accessory spermatozoa can penetrate the outer portions of the zona pellucida only, it has been thought that materials from the cortical granules must be localized or initiate modifications in some specific way in the innermost portion of the zona pellucida (Hunter, 1991a). This different penetrability of spermatozoa between outer and inner zona pellucida may be explained by different zona pellucida structures; the coarse fibrillar network in the outer zona pellucida and aggregated fibrils in the inner zona pellucida. Moreover, the presence of channel-like, radially orientated fibrils in the in vitro zygotes, detected when a pre-fixation ruthenium red and saponin treatment was used, reinforces the idea that the cortical granules are not causing a major complete change in the inner zona pellucida, as may be the case in in vivo zygotes. This finding might reflect the inability of the zona pellucida of the in vitro zygotes to prevent the entry of accessory spermatozoa to such an extent as the in vivo zygotes. However, further research is required to determine why the degree of fibrillar aggregation of zona pellucida in ovulated oocytes is much higher than that in in vitro-matured oocytes.

Funahashi et al. (2000) demonstrated that the surface morphology of the zona pellucida in both ovulated and in vitro-matured oocytes changes after sperm penetration, and that both the outer and inner zona pellucida surfaces are lysed and lose the mesh-like structure during the zona pellucida reaction. In the present study, the external meshlike structure was less apparent after sperm penetration, and fibrillar networks in both the outer and inner zona pellucida, particularly the outer network, were much more compacted after sperm penetration; however, collection of the in vitro zygotes $6-10 \mathrm{~h}$ later than their in vivo counterparts may affect the findings. These data demonstrate that the cross-sectioned morphology of zonae pellucidae in pig oocytes changes in both ovulated and in vitro-matured oocytes after sperm penetration in vivo and in vitro, respectively. However, the degree of morphological change in cross-sectioned zonae pellucidae during fertilization was much higher in ovulated oocytes than in vitro-matured oocytes. In a physiological situation, the defence mechanism against polyspermy in pig oocytes is achieved mainly by zona pellucida reaction after exocytosis of cortical granules against sperm penetration (Hunter, 1991b). However, Wang et al. (1998a) showed that the mean numbers of cortical granules beneath the plasma membrane before and after sperm penetration were not different between in vitromatured and ovulated oocytes. In the present study, complete cortical release was observed in in vitro zygotes. This evidence indicates that the differences in the morphology of cross-sectioned zonae pellucida during fertilization may be due to different zona pellucida morphology in ovulated and in vitro-matured oocytes, rather than to different amounts of cortical release. However, as induction of cortical granule exocytosis of in vitro-matured oocytes by calcium ionophore A23187 resulted in prevention of sperm penetration of the zona pellucida (Wang et al., 1998b), even the subtle morphological changes observed in in vitro zygotes in the present study may be sufficient to prevent multiple sperm penetration. However, polyspermic penetration still remains the major obstacle to successful production of large numbers of pig embryos with high levels of developmental competence by in vitro maturation and fertilization (Funahashi and Day, 1997; Day, 2000). Therefore, a delayed or incomplete zona pellucida reaction may be one of the factors that permits an abnormally high incidence of polyspermic penetration of pig oocytes in vitro.

In the present study, the mean thickness of the zona pellucida was greater in ovulated oocytes than in in vitromatured oocytes. The thickness of the zona pellucida of ovulated oocytes increased during fertilization, but did not change during IVF of in vitro-matured oocytes. These results are consistent with previous observations with SEM (Funahashi et al., 2000), despite the different processing after fixation for SEM and TEM, even when ruthenium red and saponin pre-fixation was used. As the diameter is measured by using ultrathin sections with a maximum diameter of the specimen only, our data should reflect the mean thickness of the zona pellucida. Therefore, these observations demonstrate that the zona pellucida reaction of in vitro zygotes may be far from complete. In the present study, we could not clarify whether final follicular maturation occurred in follicles just before ovulation or in the oviduct after ovulation as the cross-sectioned zona pellucida structure was not compared between follicular oocytes matured in vivo (mature oocytes in dominant follicles just before ovulation) and ovulated oocytes. Further investigation may be required to clarify the final zona pellucida maturation.

In conclusion, TEM indicates that the fine structure of the zona pellucida and the zona pellucida reaction at sperm penetration differs between pig oocytes fertilized in vivo and in vitro, and that the outer and inner pig zonae pellucidae have different network organizations, probably due to insufficient final maturation of the zona pellucida during in vitro maturation. Exposure to oviductal glycoproteins or final follicular maturation may contribute to complete maturation and induce the penetrationdependent reaction of pig zonae pellucidae. Although

Fig. 6. Transmission electron microscope images of the zona pellucida (zp) of an in vitro-fertilized pig ovum (a,c,e) and in vivo zygote $(b, d, f)$, prefixed with ruthenium red and saponin and fixed further with glutaraldehyde. $(a, b)$ The inner area of the zona pellucida is different between the (a) in vitro-fertilized and (b) in vivo zygote, with radially-orientated fibrils in (a) (arrows), that are absent in the latter (b). The higher magnification of these areas in (c) and (d) shows the clear radial versus trabecule-like orientations. The outer part of the zona pellucida partially loses these orientations (e versus f). Scale bar represents $1 \mu \mathrm{m}(\mathrm{a}, \mathrm{b})$ or $100 \mathrm{~nm}$ (c-f). 
recent developments in pig in vitro maturation systems have made it possible to produce piglets efficiently from oocytes obtained at abattoirs, further investigation is needed to reproduce the zona pellucida morphology and reaction of ovulated oocytes.

\section{References}

Brackett BG and Oliphant G (1975) Capacitation of rabbit spermatozoa in vitro. Biology of Reproduction 12 260-274

Brown CR and Cheng WKT (1986) Changes in composition of the porcine zona pellucida during development of the oocyte to the 2- to 4-cell embryo Journal of Embryology and Experimental Morphology 92 183-191

Buhi WC, O'Brien B, Alvarez IM, Erdos G and Dubois D (1993) Immunogold localization of porcine oviductal secretory proteins within the zona pellucida, perivitelline space, and plasma membrane of oviductal and uterine oocytes and early embryos Biology of Reproduction 48 1274-1283

Day BN (2000) Reproductive biotechnologies: current status in porcine reproduction Animal Reproduction Science 60-61 161-172

Dietl J and Czuppon AB (1984) Ultrastructural studies of the porcine zona pellucida during the solubilization process by Li-3,5-diiodosalicylate Gamete Research 9 45-54

Dunbar BS, Wardrip NJ and Hedrick JL (1980) Isolation, physicochemical properties, and macromolecular composition of zona pellucida from porcine oocytes Biochemistry 19 356-365

Familiari G, Nottola SA, Macchiarelli G, Micara G, Aragona C and Motta PM (1992) Human zona pellucida during in vitro fertilization: an ultrastructural study using saponin, ruthenium red, and osmiumthiocarbohydrazide Molecular Reproduction and Development 32 $51-61$

Funahashi H and Day BN (1993) Effects of the duration of exposure to supplemental hormones on cytoplasmic maturation of pig oocytes in vitro. Journal of Reproduction and Fertility 98 179-185

Funahashi H and Day BN (1997) Advances in in-vitro production of porcine embryos Journal of Reproduction and Fertility Supplement 52 271-283

Funahashi H, Cantley TC and Day BN (1994) Different hormonal requirements of porcine oocyte-complexes during maturation in vitro. Journal of Reproduction and Fertility 101 159-165
Funahashi H, Cantley TC and Day BN (1997) Synchronization of meiosis in porcine oocytes by exposure to dibutyryl cyclic AMP improves developmental competence following in vitro fertilization Biology of Reproduction $\mathbf{5 7}$ 49-53

Funahashi H, Ekwall H and Rodriguez-Martinez H (2000) Zona reaction in porcine oocytes fertilized in vivo and in vitro as seen with scanning electron microscopy Biology of Reproduction 63 1447-1452

Hedrick JL, Wardrip NJ and Berger T (1987) Differences in the macromolecular composition of the zona pellucida isolated from pig oocytes, eggs, and zygotes Journal of Experimental Zoology 241 257-262

Hunter RHF (1991a) Fertilization in the pig and horse. In A Comparative Overview of Mammalian Fertilization pp 329-349 Eds BS Dunbar and MG O'Rand. Plenum Press, New York

Hunter RHF (1991b) Oviduct function in pigs, with particular reference to the pathological condition of polyspermy Molecular Reproduction and Development 29 385-391

Kim N-H, Funahashi H, Abeydeera LR, Moon SJ, Prather RS and Day BN (1996) Effects of oviductal fluid on sperm penetration and cortical granule exocytosis during in vitro fertilization of porcine oocytes Journal of Reproduction and Fertility 107 79-86

Petters RM and Wells KD (1993) Culture of pig embryos Journal of Reproduction and Fertility Supplement 48 61-73

Wang WH, Abeydeera LR, Prather RS and Day BN (1998a) Morphologic comparison of ovulated and in vitro-matured porcine oocytes, with particular reference to polyspermy after in vitro fertilization Molecular Reproduction and Development 49 308-316

Wang WH, Machaty Z, Abeydeera LR, Prather RS and Day BN (1998b) Parthenogenetic activation of pig oocytes with calcium ionophore and the block to sperm penetration after activation Biology of Reproduction 58 1357-1366

Wang WH, Machaty Z, Abeydeera LR, Prather RS and Day BN (1999) Time course of cortical and zona reaction of pig oocytes upon intracellular calcium increase induced by thimerosal Zygote 7 79-86

Received 3 April 2001

First decision 1 May 2001

Accepted 22 May 2001. 